\title{
Author Index to Abstracts
}

\author{
(Numbers cited refer to abstract numbers)
}

\author{
Abreu S, 21 \\ Aebert E, 3 \\ Alé $\mathrm{G}, 23$ \\ Ambiela R, 11 \\ Aranda E, 19 \\ Arata M, 33 \\ Arnhold IJP, 15, 20, 25, 37 \\ Asenjo S, 1 \\ Balzaretti M, 28 \\ Barrera A, 14 \\ Basabe JC, 17, 33, 34 \\ Beas F, 22, 27 \\ Belgorosky A, 8, 18, 32, 40 \\ Bergadá C, 2, 3, 4, 6, 7, 10, 12, 19 , \\ $29,31,32,35$ \\ Bergadá I, 3, 4, 29, 31 \\ Bianco AC, 25 \\ Bloise W, 15, 20, 37 \\ Boguszewsky MS, 13, 36 \\ Boquete $\mathrm{H}, 11,16$ \\ Boric MA, 27 \\ Boulgourdjian E, 35 \\ Bugueño M, 22 \\ Cabut V, 16 \\ Cassinelli HR, 12 \\ Cassorla F, 22 \\ Castellano M, 8 \\ Cattani A, 22 \\ Chaler E, 40 \\ Charler E, 8 \\ Chemes $\mathrm{H}, 6$ \\ Chiesa A, 4, 30 \\ Ciaccio M, 18, 40 \\ Coarconi SM, 37 \\ Coco R, 10 \\ Copelli S, 10
}

Corah D, 10

Cortinez A, 14, 22

D'Agostino D, 28

de Lacerda Filho L, 13

De Lacerda L, 36

del Rey G, 10

Doval A, 17

Eggers M, 14

Escobar Maria E, 35

Escobar ME, 32

Estefan V, 25

Fabiano de Bruno L, 17

Fainstein-Day F, 28

Fernández E, 26

Fideleff $\mathrm{H}, 11,16$

García H, 14, 22, 38, 39

García Rudaz C, 19

Gleisner A, 1

Goldberg J, 9

Goto SY, 20

Gottlieb S, 6

Granillo E, 28

Gruñeiro L, 4, 30

Gryngarten M, 32

Guazzeli ICM, 25

Gutman RA, 28

Heinrich JJ, 3, 7, 19, 31, 35

Henríquez $\mathrm{C}, 22$

Herzovich V, 9

Holland M, 11, 16

Iñiquez $\mathrm{G}, 27$

Iorcansky S, 9
Jara A, 22

Jasper H, 21

Kaplan R, 23

Karabatas L, 34

Keselman A, 7, 31

Kohara SK, 36

Kohek MBF, 15

Lando VS, 15, 20

Libman J, 24

Llera J, 28

Lombardo Y, 34

Maceiras M, 8, 40

Martínez A, 2, 7, 19, 31, 35

Mautalén C, 12

Medel R, 6

Mendoça B, 20

Mendoça BB, 15, 25, 37

Menichini A, 24

Miglietta A, 24

Miras M, 23

Muños L, 5

Muñoz MS, 23

Nicolau W, 25, 37

Ortiz E, 26

Osinde E, 17

Osorio MGF, 25, 37

Paez A, 23

Pagano SM, 16

Panteón E, 14, 38, 39

Pasqualini T, 28

Perco M, 11

Péres de Rubio G, 5

Pérez E, 27
Pérez V, 14

Podestá M, 6

Quesada E, 6

Quintanas C, 33

Raizman $\mathrm{H}, 4$

Rigon AC, 15, 20

Rivarola MA, 8, 18, 40

Rojas S, 1

Ropelato G, 2, 6

Rossi J, 9, 28

Sandrini R, 13, 36

Sasky N, 16

Saskyn N, 11

Schmitt Lobe MN, 36

Segura TC, 25

Silva R, 22

Sobrado P, 11

Targovnick, 10

Téllez R, 38

Testa L, 23

Torrealba I, 14, 22, 38, 39

Trifone L, 4

Turconi A, 8

Ugarte F, 14

Vaconcelos C, 20

Vasconcelos C, 15

Venegas G, 1

Vildósola B, 38

Wilhelm V, 1, 22

Yanicowsky ML, 26

Youlton R, 22

Zanchetti F, 11 